\title{
Resazurin as an indicator of reducing capacity for analyzing the physiologic status of deep-sea bacterium Photobacterium phosphoreum ANT-2200
}

Jie Dai, Hongzhi Tang, Xuegong Li, Claire-Lise Santini, Wenpeng Cui, Na Liu, Xiaoqing Qi, Xuehua Cui, Alain Grossi, Philippe Notareschi, et al.

\section{To cite this version:}

Jie Dai, Hongzhi Tang, Xuegong Li, Claire-Lise Santini, Wenpeng Cui, et al.. Resazurin as an indicator of reducing capacity for analyzing the physiologic status of deep-sea bacterium Photobacterium phosphoreum ANT-2200. Chinese Journal of Oceanology and Limnology, Springer Verlag, In press, 10.1007/s00343-020-9301-8 . hal-02999423

\section{HAL Id: hal-02999423 \\ https://hal.archives-ouvertes.fr/hal-02999423}

Submitted on 20 Dec 2020

HAL is a multi-disciplinary open access archive for the deposit and dissemination of scientific research documents, whether they are published or not. The documents may come from teaching and research institutions in France or abroad, or from public or private research centers.
L'archive ouverte pluridisciplinaire HAL, est destinée au dépôt et à la diffusion de documents scientifiques de niveau recherche, publiés ou non, émanant des établissements d'enseignement et de recherche français ou étrangers, des laboratoires publics ou privés. 
Using resazurin as a reducing capacity indicator for analyzing physiological status of deep-sea bacterium Photobacterium phosphoreum ANT-2200

DAI Jie ${ }^{1,2,3}$, TANG Hong-Zhi ${ }^{1,2,3}$, LI Xue-Gong ${ }^{1,3}$, SANTINI Claire-Lise ${ }^{3,4}$, CUI Wen-Peng ${ }^{1,2,3}$, LIU Na ${ }^{1,2,3}$, QI Xiao-Qing ${ }^{1,3}$, CUI Xue-Hua ${ }^{1,3}$, GROSSI Alain ${ }^{5}$, NOTARESCHI Philippe ${ }^{4}$, ZHANG Wei-Jia ${ }^{1,3 * *}$ and WU Long-Fei ${ }^{3,4^{* *}}$

1 Laboratory of Deep Sea Microbial Cell Biology, Institute of Deep Sea Science and Engineering, Chinese Academy of Sciences, Sanya, 572000, China.

2 University of Chinese Academy of Sciences, Beijing 100864, China.

3 International Associated Laboratory of Evolution and Development of Magnetotactic Multicellular Organisms (LIA-MagMC), CNRS-CAS

4 Aix Marseille University, CNRS, LCB, IMM, FR3479, IM2B, Marseille, 13402, France.

5 Aix Marseille University, CNRS, IMM, FR3479, Marseille, 13402, France.

* For correspondence:

Long-Fei WU, wu@imm.cnrs.fr; 33-4 91164157

Wei-Jia ZHANG, wzhang@idsse.ac.cn; 86-0898 88211771 


\begin{abstract}
Resazurin (RZ) is a weak fluorescent blue dye and can be irreversibly reduced to highly fluorescent pink colored resorufin (RF), and further reversibly reduced to colorless dihydroresorufin (hRF) by photo-deoxygenation, chemical reaction and reductive organic compounds produced through cell metabolism. Because of the reliable and sensitive fluorescence-color change and noninvasive features, resazurin has been widely used as a redox indicator in cell viability and proliferation assays for bacteria, yeast and mammalian cells. However, resazurin is rarely used in physiological characterization of marine microorganisms. Here we developed a custom-made irradiation and absorption analysis device to assess reducing capacity and physiological status of marine bacterial cultures. We measured the absorption spectra of $\mathrm{RZ}, \mathrm{RF}$ and $\mathrm{hRF}$ in presence of reducing compound $\mathrm{Na}_{2} \mathrm{~S}$ and under visible light irradiation. After establishing the appropriate parameters, we monitored the color changes of resazurin and its reduced derivatives to evaluate the coherence between reducing capacity, bioluminescence and growth of deep-sea bacterium Photobacterium phosphoreum ANT-2200 under various conditions. Emission of bioluminescence is an oxidation process that depends on cellular reducing capacity. We found that growth and bioluminescence of ANT-2200 cultures were progressively impeded with increased concentration of RZ, which suggests a competition for reducing molecules between RZ at high concentration with reductive metabolism. Therefore, it should be cautious when RZ is added directly in growth media to monitor the culture redox. Analysis of RZ reduction velocity of ANT-2200 cultures showed a detrimental effect of high hydrostatic pressure and revealed a high coherence between the reducing capacity and bioluminescence of the cultures. This study clearly demonstrates the potential of using resazurin to characterize microbial metabolism and physiology of marine bacteria.
\end{abstract}

Keywords: Oxic-reduction indicator, absorption spectra, bacterial growth, marine microorganism

\title{
1 INTRODUCTION
}

Resazurin (RZ) has a chemical structure as 7-hydroxy-10-oxidophenoxazin-10-ium-3-one and is also referred to as heterocyclic $N$-oxide or N-phenoxazine-3-one dye. Resazurin exhibits a blue color with weak fluorescence. As a donor in oxygen atom transfer reaction, RZ is irreversibly reduced to highly fluorescent pink dye resorufin $(\mathrm{RF})$. Further reduction of RF results in the production of colorless dihydroresorufin (hRF), which is a reversible process (Fig. 1). Resazurin is water soluble, noninvasive, low or noncytotoxic. Therefore, resazurin and derivatives of N-phenoxzine-3-one dyes have been widely used as a simple, rapid, reliable, sensitive, safe and 
cost-effective measurement of cell viability and proliferation, and determination of bacterial or yeast contamination in milk (Kowaltowski, 2019; Rampersad, 2012; Neumann, et al., 1996). Moreover, the anionic dye RZ is also appropriate probe for analytical determination of ions such as $\mathrm{Se}(\mathrm{IV})$ and $\mathrm{Pb}(\mathrm{II})$ (Safavi, et al., 1990; Afkhami, et al., 1991). However, the resazurin dyes remain poorly utilized in physiological studies of marine microorganisms.

a

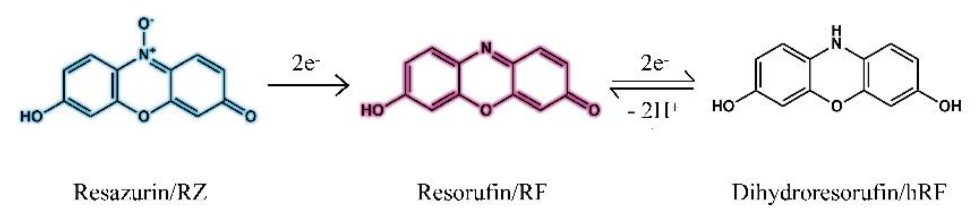

b

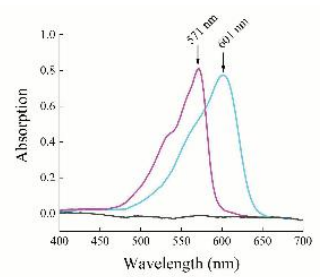

Fig. 1 Molecular structure and absorption spectra of resazurin, resorufin and dihydroresorufin. (a) illustrates reduction of resazurin (RZ, blue color) to resorufin (RF, pink) and further to colorless dihydroresorufin (hRF). (b) shows the absorption spectra of RZ (cyan curve), RF (red color) and hRF (black color). The absorption spectra were obtained in this study.

Reduction of the phenoxazine-3-one dyes, RZ and RF occurs through chemical reaction and metabolism process. In addition, irradiation in the presence of amines also leads to deoxygenation of RZ and its conversion to RF (Neumann, et al., 1996; Bueno, et al., 2002). Similarly, reduction of RF is a photosensitive reaction. Zhao et al., have reported that UV and visible light $(\lambda>300 \mathrm{~nm})$ irradiation leads to one-electron reduction of RF and produces a semiquinoneimide-type anion radical $\mathrm{RF}^{*-}$ under anaerobic conditions with reduced nicotinamide adenine dinucleotide (NADH) (Zhao, et al., 2011). Continuous irradiation further reduces $\mathrm{RF}^{*-}$ to its colorless form of dihydroresorufin (Fig. 1).

Bioluminescent bacteria have the peculiar capacity of emitting blue-green light $(470 \sim 490 \mathrm{~nm})$ through the luciferase-catalyzed oxidation of reduced flavin mononucleotide (FMNH) and fatty aldehyde (Fig. 2) (Dunlap \& Kita-Tsukamoto, 2006). To continue the bioluminescent process, oxidized FMN must be reduced to FMNH by an enzyme using NADH as electron donor (Fig. 2). Most of the luminous bacteria dwell in marine environments from the shallow coast to deep-sea (Dunlap \& Kita-Tsukamoto, 2006). The Photobacterium phosphoreum strain ANT-2200 (hereafter 
called ANT-2200) was isolated at $2200 \mathrm{~m}$ depth from the Mediterranean Sea and capable of emitting bioluminescence (Al Ali et al., 2010; Martini et al., 2013). Genomic and physiological analyses revealed a versatile energy metabolic potential and growth capacity of ANT-2200 by deriving energy from fermentation of glucose or maltose, by respiration with formate as electron donor and trimethlyamine N-oxide (TMAO), nitrate or fumarate as electron acceptors, or by chemo-organo-heterotrophic growth in rich media (Zhang, et al., 2016). Interestingly, the light emission seems to be proportional with the growth rate. Continuous emission of bioluminescence depends on cell reductive capacity and the bio-luminous light might trigger the photo-reduction of $\mathrm{RZ}$ and RF. We wondered if it is feasible to use resazurin dyes to assess physiological status of ANT-2200 by analyzing the correlation among the reducing capacity, bioluminescence and growth. We set up an irradiation and spectrum-scanning device and showed the reliable coherence of RZ reduction with the growth and luminescence of ANT-2200. This study demonstrated using the heterocyclic $N$-oxide dyes in the physiological and metabolic investigation of marine microorganisms.

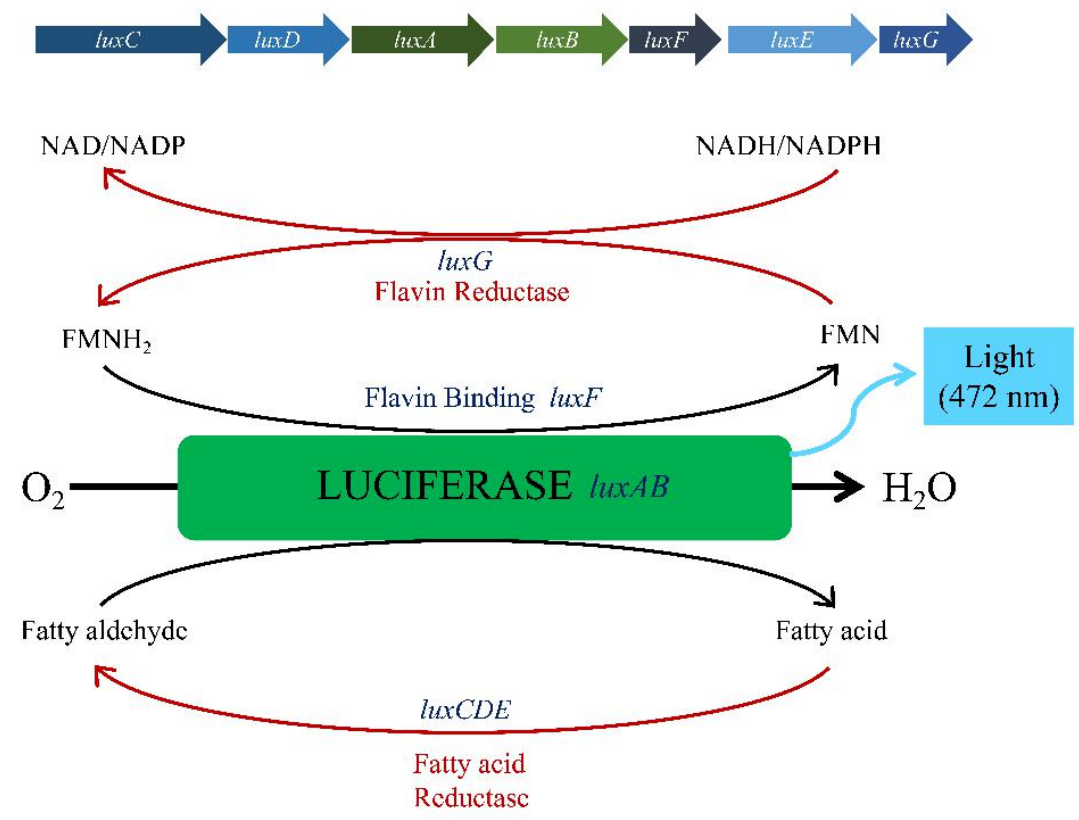

Fig. 2. Bioluminescence of strain ANT-2200. The $l u x$ gene cluster of ANT-2200 contains the genes coding the enzymes required for the bioluminescence process of ANT-2200 and regeneration of reduced substrates. 


\section{MATERIALS AND METHODS}

\subsection{Strain and bacterial cultures}

Cultures of the strain ANT-2200 were performed in YPG media at $27{ }^{\circ} \mathrm{C}$ in serum bottles (Fig. S1), or in $5 \mathrm{ml}$ syringes immerged in high-pressure incubators as described by Zhang et al. (Zhang, et al., 2016). When indicated, 20 MPa hydrostatic pressure was applied on high pressure incubators that contain the syringes. A stock solution of $20 \mathrm{mM}$ resazurin was prepared in Milli-Q water and used at final concentrations as indicated.

To analyze effect of irradiation, resazurin solutions or bacterial cultures were exposed to tungsten bulb or $470 \mathrm{~nm}$ LED. Light densities were measured using Thorlabs S120ve sensor connected to Thorlabs powermeter.

Samples were taken at indicated time, and optical density at $600 \mathrm{~nm}$ was measured using spectrophotometer and luminescence was analyzed using microplate reader according to fabricant instructions.

\subsection{Absorption spectra analysis}

Absorption spectra of resazurin and its reduced derivatives were measured in serum bottles, directly as solution or in growth media at the concentrations as indicated. Alternatively for post-growth scanning, 7 to $8 \mathrm{ml}$ cultures were transferred to serum bottles and resazurin was added at $0.01 \mathrm{mM}$ final concentration, the bottles were sealed and scanned for the spectra every 5 min until the end of color change using Varian 50 or Agilent Technologies Cary60 UV-Vis spectrophotomers. Bottle holder was designed and fabricated by 3D-printing (Fig. S1).

\section{RESULTS AND DISCUSSION}

\subsection{Spectral characteristics of resazurin and its derivatives}

3.1.1 Set-up of a custom-made device to facilitate irradiation and spectrum scanning of cultures Because RZ and RF are highly sensitive to oxidation, tight sealed containers should be used for bacterial cultivation and absorption spectra analysis. The $11 \mathrm{ml}$ serum bottles are ideal for this study for following reasons: first of all, they can be easily tight sealed and addition of chemical solutions or exchange of the gases can be performed using needles piercing the rubber plugs; secondly, culturing marine bacteria, such as Photobacterium phosphoerum ANT-2200, with these bottles can provide enough biomass for further analysis; and most importantly, the bottle fits in 
most standard spectrophotometers. To use these bottles with Varian 50 and Agilent Technologies Cary60 UV-Vis spectrophotomers, we designed a 3-D printed bottle holder for directly scanning the absorption spectra in these bottles (Fig S1). In addition, LED light bulbs can be easily mounted on the holders to irradiate the solution or bacterial cultures.

Using the bottle-Cary60 scanning device we observed a principal absorption at $601 \mathrm{~nm}$ and a shoulder peak at $550 \mathrm{~nm}$ of RZ in water solution saturated with nitrogen gas (Fig. 1b). Reduction of $\mathrm{RZ}$ to $\mathrm{RF}$ by $\mathrm{Na}_{2} \mathrm{~S}$ resulted in a shift of the spectrum with the major peak at $571 \mathrm{~nm}$ and shoulder peak around $530 \mathrm{~nm}$ (Fig. 1b). Further reduction to hRF led to disappearance of the absorption peaks. The absorption spectra of RZ, RF and hRF are similar with previous reports, although slight shift of absorption peaks that possibly caused by different media and spectrophotometers were observed. Therefore, this device allows us easily monitoring the reduction states of RZ, RF and hRF in sealed serum bottles, and peaks at $601 \mathrm{~nm}$ and $571 \mathrm{~nm}$ were used to represent RZ and RF, respectively, in this study.

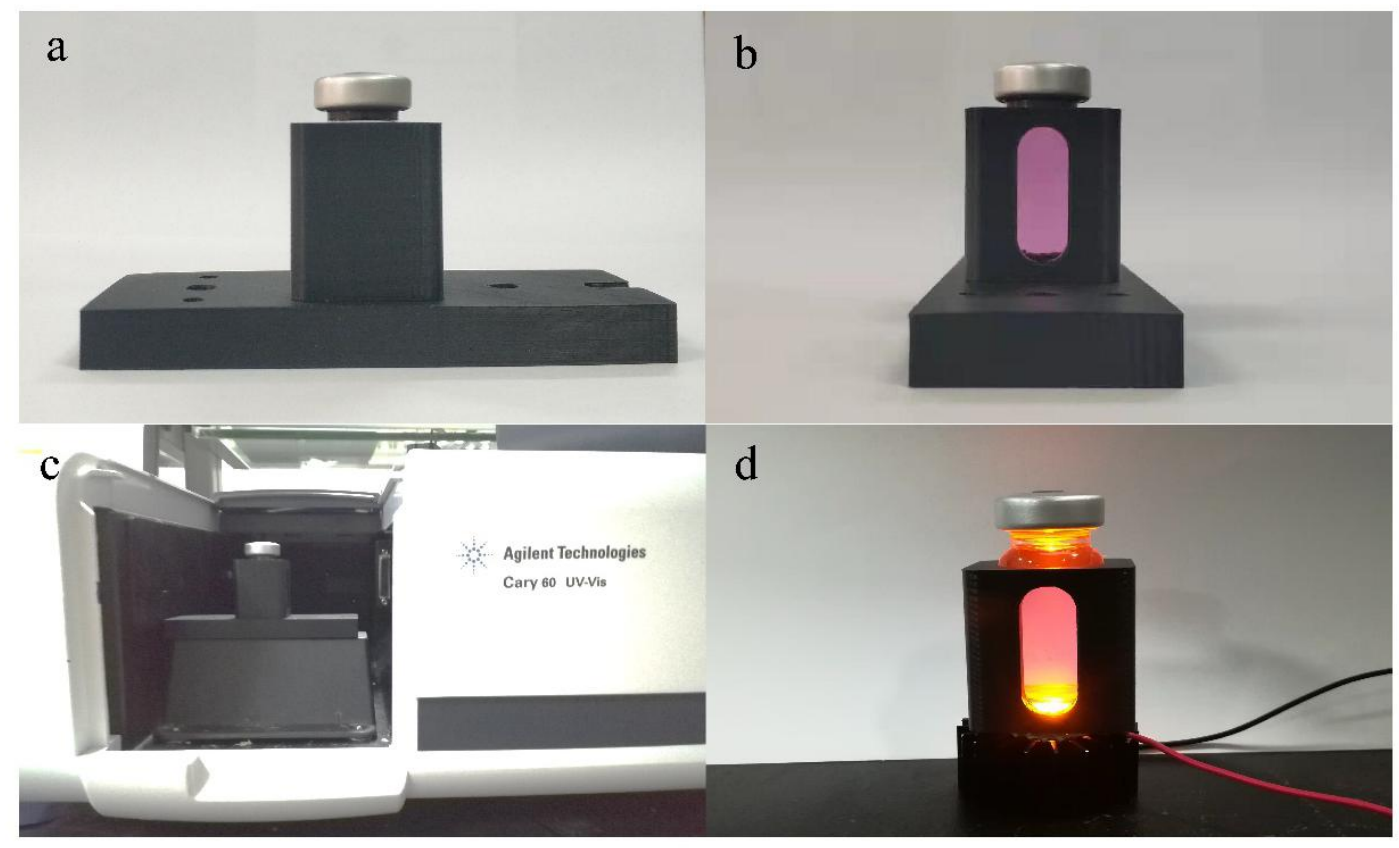

Fig S1 Bottle-scanning device for spectra scanning and irradiation in serum bottles. The 3-D printed bottle holders can be used for real-time scanning of the culture absorption spectra (a-c) and irradiation of cultures with LEDs at different wavelengths (d). 
3.1.2 Effect of irradiation and $\mathrm{Na}_{2} \mathrm{~S}$ on the absorption spectra of resazurin and its derivatives

We first assessed the color change of RZ under different redox conditions in artificial seawater in a fermenter with controlled redox and $\mathrm{pH}$. The solution had a blue color and remained unchanged after over-night bubbling with nitrogen gas. Changing the $\mathrm{N}_{2}$ gas to carbon dioxide increased the redox potential from $-90 \mathrm{mV}$ to $-53 \mathrm{mV}$ and decreased $\mathrm{pH}$ of the solution from 8.9 to 7.9 , but had no effect on the blue color. Therefore, the RZ solution is stable in the ranges of redox potential and $\mathrm{pH}$ under the conditions used. Decreasing solution redox along seems not sufficient to convert RZ to RF.

Other than $\mathrm{pH}$ and redox condition, irradiation has been reported leading to deoxygenation of RZ and reduction of RF under certain conditions (Porcal, et al., 2011). Consistently we found that when exposed to sunlight or illuminated with a tungsten light bulb, the solution became light-pink color, which implies the formation of RF from photochemical deoxygenation of RZ under this redox and $\mathrm{pH}$ condition in artificial seawater solution.

We then evaluated effect of irradiation and chemical reaction using the bottles-scanning devices. The $11 \mathrm{ml}$ serum bottles were filled up with $8 \mathrm{ml}$ water solution containing $7.2 \mu \mathrm{M} \mathrm{RZ}$ and bubbled with $\mathrm{N}_{2}$ for $15 \mathrm{~min}$. Then $\mathrm{Na}_{2} \mathrm{~S}$ were injected in the bottles to final concentrations at 72 $\mu \mathrm{M}$ (Fig. S2, bottle 1 and 6), $720 \mu \mathrm{M}$ (bottles 2 and 7), $3600 \mu \mathrm{M}$ (bottles 3 and 8) and $7200 \mu \mathrm{M}$ (bottles 4 and 9). As a control the bottles 5 and 10 contained only RZ without $\mathrm{Na}_{2} \mathrm{~S}$. The bottles were incubated in dark (bottles 1-5) or with illumination from tungsten light bulb (bottles 6-10, at $25 \mathrm{~mW} / \mathrm{cm}^{2}$ ). Visual observation showed that $\mathrm{RZ}$ remained blue color in the absence of $\mathrm{Na}_{2} \mathrm{~S}$ (Fig. S2a). At the 10-fold molar ratio of $\mathrm{Na}_{2} \mathrm{~S} / \mathrm{RZ}$ (bottles 1 and 6 ) the solutions turned to pink color (RF), whereas all solutions became colorless (hRF) with the molar ratio of $\mathrm{Na}_{2} \mathrm{~S} / \mathrm{RZ}$ higher than 100-fold. 
a

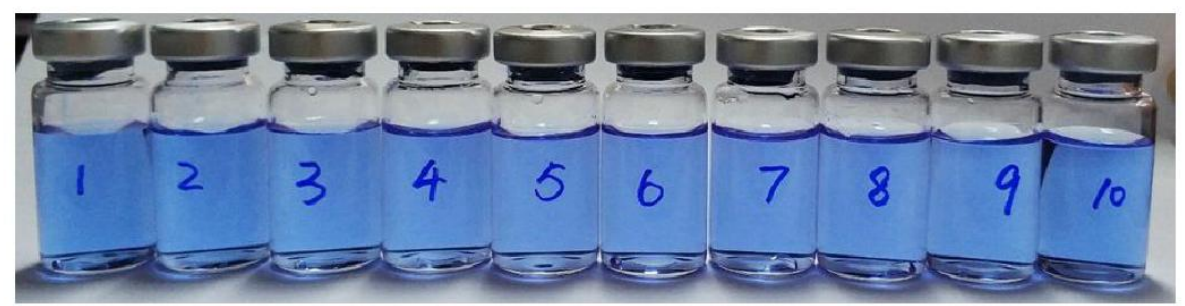

b

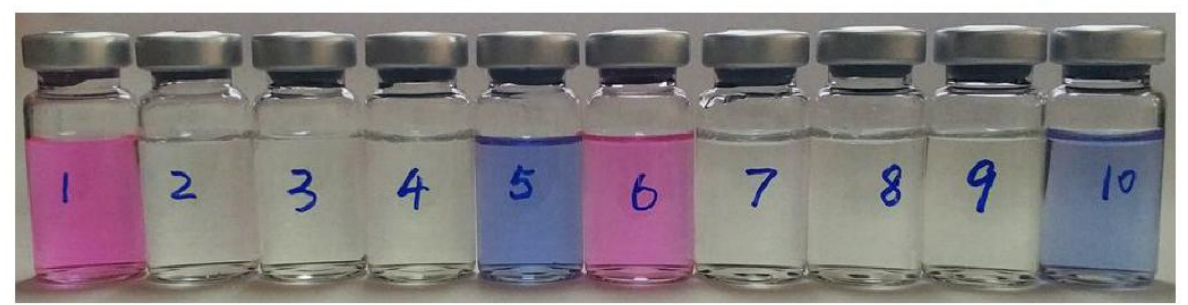

Fig. S2. Reduction of the phenoxazine-3-one dyes by chemical reaction and irradiation. The $11 \mathrm{ml}$ bottles were filled up with $8 \mathrm{ml}$ water solution containing 7.2 $\mu \mathrm{M} \mathrm{RZ}$ and bubbled with $\mathrm{N}_{2}$ for 15 min (a). Then $\mathrm{Na}_{2} \mathrm{~S}$ were injected in the bottles to final concentrations of $72 \mu \mathrm{M}$ (b, bottle 1 and 6), $720 \mu \mathrm{M}$ (b, bottles 2 and 7), 3600 $\mu \mathrm{M}$ (b, bottles 3 and 8 ) and $7200 \mu \mathrm{M}$ (b, bottles 4 and 9). As a control the bottles 5 and 10 contain only RZ without $\mathrm{Na}_{2} \mathrm{~S}(\mathrm{~b})$. The bottles were incubated in dark (1-5) or with irradiation (6-10, tungsten light bulb at 25 $\left.\mathrm{mW} / \mathrm{cm}^{2}\right)$

We further quantified the effect of reductant and irradiation by monitoring the time-lapse of absorption spectra. As shown in Fig. 3, the spectra of RZ remained unchanged during 24 hours in the absence of $\mathrm{Na}_{2} \mathrm{~S}$ and light (Fig. 3a). However, the spectra diminished slightly or substantially after $12 \mathrm{~h}$ and $24 \mathrm{~h}$ irradiation with tungsten light, respectively (Fig. $3 \mathrm{~b}$ ). Notably, the scanning revealed spectral change, which was unperceivable by naked-eye. Therefore, the irradiation led to photobleaching and photo-deoxygenation of the RZ as reported by Porcal et al. (Porcal, et al., 2011).

With addition of $\mathrm{Na}_{2} \mathrm{~S}$, decrease of $\mathrm{RZ}$ was concomitant with rise of RF spectra, and the decline of the latter afterwards under certain circumstances. The rates of spectral changing were in proportion with molarities ratio of $\mathrm{Na}_{2} \mathrm{~S} / \mathrm{RZ}$ (Fig. 3c-f). When incubated in darkness, addition of ten-fold amount of $\mathrm{Na}_{2} \mathrm{~S}$ led to decrease of absorption at $601 \mathrm{~nm}$ from 0.78 to 0.33 and increase of that at $571 \mathrm{~nm}$ from 0.54 to 0.82 within $96 \mathrm{~min}$ (Fig. 3c and g). Notably, the absorption at $571 \mathrm{~nm}$ kept increasing under this condition (Fig. 3g dash-red curve), indicating RF was not further reduced into $\mathrm{hRF}$ within the analyzing time. The reduction process of RZ-RF-hRF can be 
accelerated by irradiation. When exposed to irradiation, the peak at $601 \mathrm{~nm}$ decreased quickly to baseline level at $15 \mathrm{~min}$ (Fig. $3 \mathrm{~d}$ and g, blue curve), when absorption at $571 \mathrm{~nm}$ reached its maximum and began to decrease gradually (Fig. $3 \mathrm{~g}$, red curve). By the end of analysis at $40 \mathrm{~min}$, $\mathrm{RF}$ was partially reduced into transparent $\mathrm{hRF}$ that led to both lower absorption at $571 \mathrm{~nm}$ and a lighter pink color of the solution (Fig. 3d and Fig. S2, bottle 6).

As demonstrated above, the amount of reductant had great influence of the reduction of RZ. When excessive $\mathrm{Na}_{2} \mathrm{~S}$ (molar ratio of $\mathrm{Na}_{2} \mathrm{~S} / \mathrm{RZ}$ of 500 ) was added, the $601 \mathrm{~nm}$ absorption decreased quickly to the baseline level in approximate $1 \mathrm{~min}$ (Fig. 3e, f and blue curves in h). The $571 \mathrm{~nm}$ absorption increased to the maximal level at $30 \mathrm{~s}$, and then decreased to the approximate baseline level at $2.5 \mathrm{~min}$ after the addition of $\mathrm{Na}_{2} \mathrm{~S}$, respectively. Irradiation did not change the reduction velocity (Fig. 3h), which indicates a dominant chemical reduction effect over the irradiation at excessive amount of $\mathrm{Na}_{2} \mathrm{~S}$.

Altogether, these results show that $\mathrm{Na}_{2} \mathrm{~S}$ chemically reduces $\mathrm{RZ}$ to $\mathrm{RF}$ and $\mathrm{hRF}$, and irradiation with visible light leads to photo-deoxygenation of $\mathrm{RZ}$ and accelerates the reduction process in the bottle-scanning systems. Evolution of the absorption peak at $571 \mathrm{~nm}$ was resulted from reduction of RZ in combination with further reduction of RF to hRF. In contrast, changes of absorption at $601 \mathrm{~nm}$ reflects the irreversible reduction of $\mathrm{RZ}$ alone, and therefore could be a better indicator of reducing capacity in the reaction system. In order to quantify and compare the reducing capacity between different samples, instantaneous reduction velocity was calculated using $\mathrm{V}_{\mathrm{n}}=d\left(\mathrm{~A}_{601}{ }_{\mathrm{n}}{ }^{+1}\right.$ $\left.\mathrm{A}_{601 \_n} \mathrm{n}\right) / d\left(\mathrm{t}_{-} \mathrm{n}+1-\mathrm{t}_{-} \mathrm{n}\right)$, where $\mathrm{A}_{601 \_n}$ and $\mathrm{t}_{-} \mathrm{n}$ are the absorption at $601 \mathrm{~nm}$ and the time at point $\mathrm{n}$, respectively. As shown in Fig. 3, the instantaneous reduction velocity increased from 0.005 to $0.08 \mathrm{OD}_{601} / \mathrm{min}$ upon illumination at limited amount of $\mathrm{Na}_{2} \mathrm{~S}(\mathrm{~g})$, whereas it reached the maximal 1.04 and $1.05 \mathrm{OD}_{601} / \mathrm{min}$ at excessive $\mathrm{Na}_{2} \mathrm{~S}$ independently of illumination (h). The $\mathrm{V}_{\mathrm{n}}$ will be used to describe reducing capacity of the cultures in this study. 
a

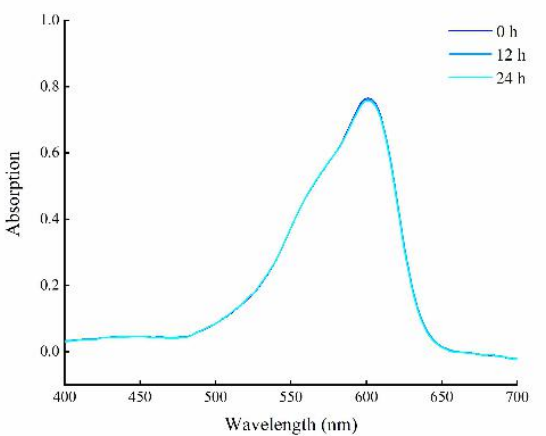

$\mathrm{c}$

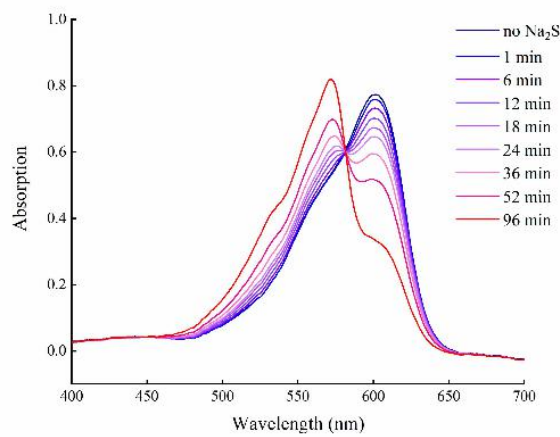

$\mathrm{e}$

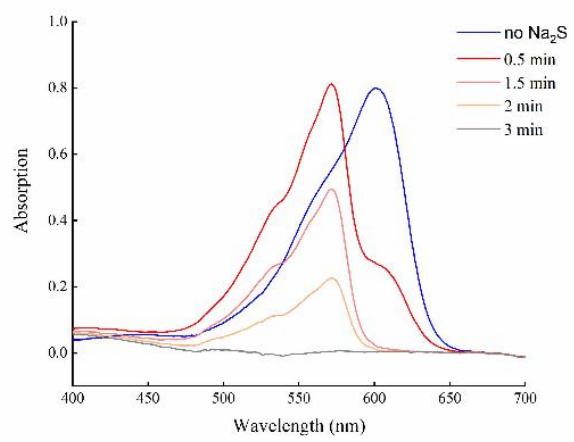

$\mathrm{g}$

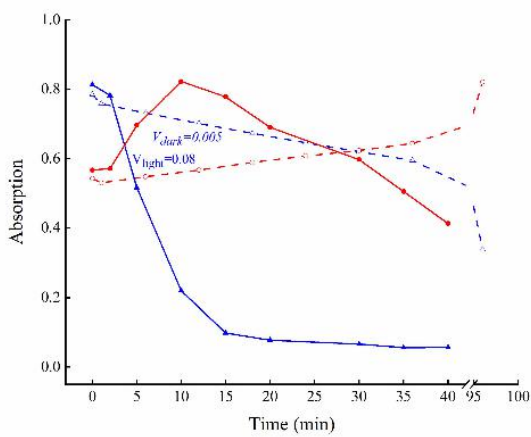

$\mathrm{b}$

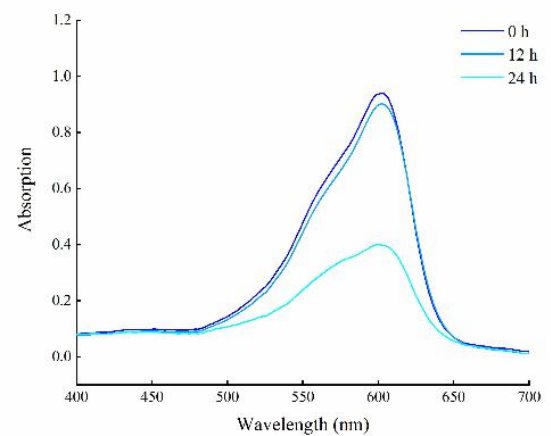

d

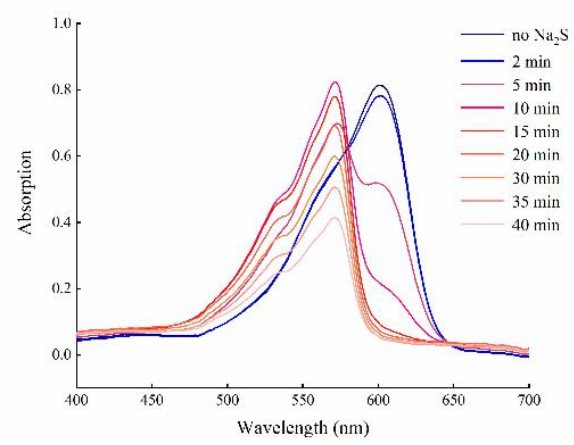

f

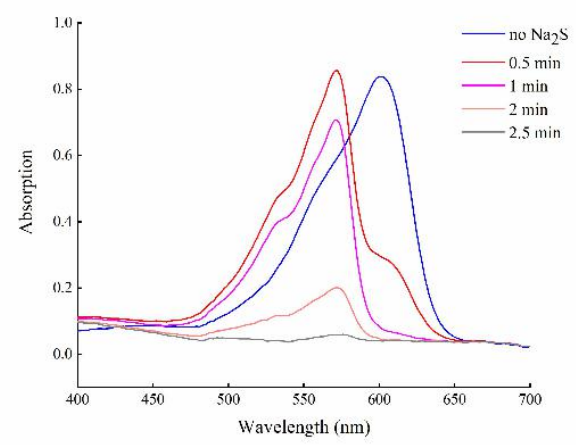

$\mathrm{h}$

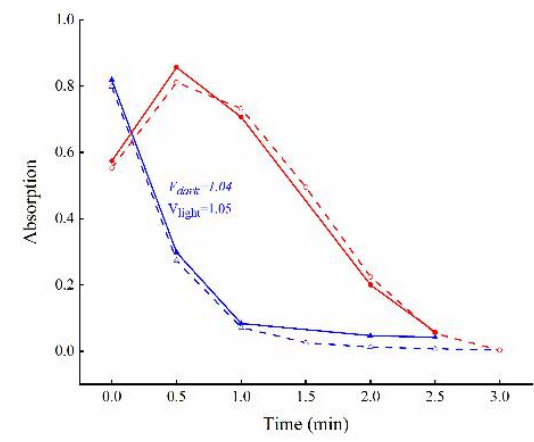

Fig. 3 Effect of $\mathrm{Na}_{2} \mathrm{~S}$ and irradiation on absorption spectra of resazurin, resorufin and dihydroresorufin. RZ solutions without $\mathrm{Na}_{2} \mathrm{~S}\left(\mathrm{a}\right.$ and $\mathrm{b}$ ) or with $\mathrm{Na}_{2} \mathrm{~S} / \mathrm{RZ}$ molar ratio of 10:1 (c and d) and 500:1 (e and f) were scanned with time interval as indicated. $\mathrm{g}$ and $\mathrm{h}$ show the changes of the RZ-601 nm (blue curves) and RF-571 $\mathrm{nm}$ (red curves) of RZ solutions with 10:1 (g) and 500:1 (h) $\mathrm{Na}_{2} \mathrm{~S} / \mathrm{RZ}$ molar ratio without (dash lines) or with (lines) 
irradiations. The maximal instantaneous reduction velocities are indicated in $\mathrm{g}$ and $\mathrm{h}$.

\subsection{Using reducing capacity indicator RZ to evaluate physiological status of ANT-2200}

\subsubsection{Effect of resazurin on the growth of ANT-2200}

Bioluminescent bacteria emit light by a well conserved FMNH oxidation reaction (Fig. 2). To continue the emission, the oxidized FMN must be reduced back to FMNH by NADH or NADPH, which is catalyzed by flavin reductase LuxG in marine luminous bacteria (Nijvipakul et al., 2008). In parallel resazurin is converted to resorufin by reductant NADPH or NADH in the presence of the enzyme NADPH dehydrogenase or NADH dehydrogenase in living cells (Jong \& Woodlief, 1977; Barnes \& Spenney, 1980). Therefore, resazurin might compete with LuxG for the reductant NADPH/NADH and affect bioluminescence. To assess this hypothesis, we analyzed the effect of addition of resazurin on the culture of ANT-2200. Interestingly we observed that the growth of ANT-2200 was progressively hampered with increased resazurin in the growth media (Fig. 4a). Moreover, the bioluminescence was parallelly decreased (Fig. 4b).

We then analyzed the reducing capacity of these cultures. Since the resazurin in the growth media was irreversibly converted into RF during the growth of ANT-2200 cells, we followed the changes in characteristic RF absorption peak at $571 \mathrm{~nm}$ instead. By shaking the culture bottles, hRF generated during the growth was re-oxidized into RF by the air phase in the culture bottles. Then RF would be reduced into hRF by ANT-2200 cells. By measuring time-lapse absorption spectra we would be able to compare the rates and extents of RF reduction that reflect the physiological status of these cultures. As shown in Figure 4c, absorption at $571 \mathrm{~nm}$ of the culture with $0.01 \mathrm{mM}$ RZ dropped to the baseline level 20 min after shaking. However, it took 30 min for the RF peak reduced to approximate half with cells cultured with $0.05 \mathrm{mM} \mathrm{RZ} \mathrm{(Fig.} \mathrm{4d).} \mathrm{These} \mathrm{results} \mathrm{indicate}$ that $0.05 \mathrm{mM} \mathrm{RZ}$ might be present at excessive amount to be completely reduced by the cells. The excessive amount of RZ in the media might also account for its inhibition effect of on the growth and bioluminescence. Therefore, caution should be taken when RZ is added in media to monitor the redox of cultures. 
a

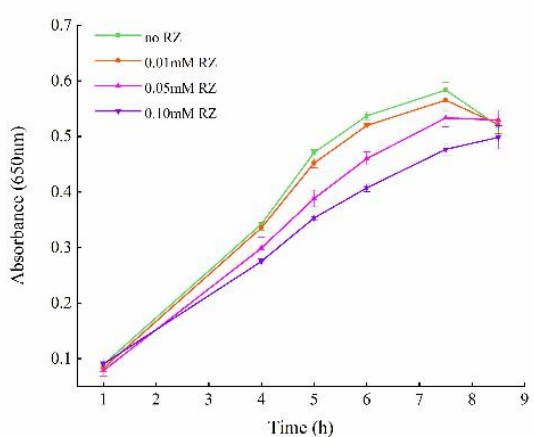

c

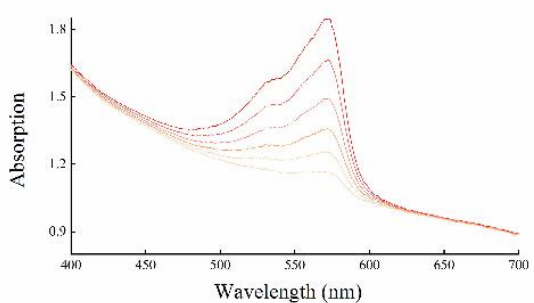

b

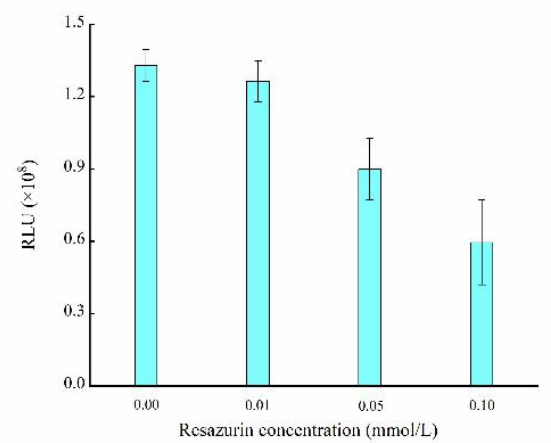

d

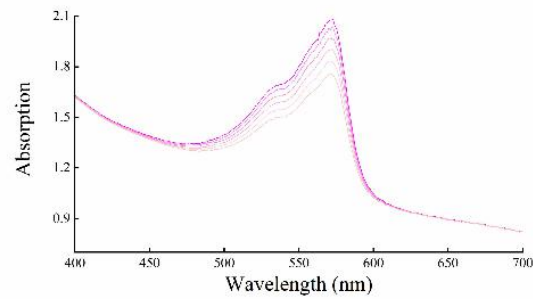

Fig. 4 Influence of RZ on ANT-2200 growth and bioluminescence. RZ was added in the cultures bottles at concentrations as indicated. The optical densities of the cultures (a) were measured at times indicated. At the end of the incubation bioluminescence was detected (b), and the reducing capacities of cultures with $0.01 \mathrm{mM}(\mathrm{c})$ and $0.05 \mathrm{mM}$ (d) RZ were analyzed by scanning the absorption spectra with 5 min intervals after shaking the bottles to re-oxidize hRF to $\mathrm{RF}$.

3.2.2 Coherence of using bioluminescence and instantaneous reducing velocity to analyze physiological state of ANT-2200 cultures

The deep-sea luminous strain ANT-2200 has versatile energy metabolic potential (Zhang, et al., 2016) and its light emission seems to be proportional with growth rate. We thought to assess the proof of concept of using resazurin to evaluate the reducing capacity of marine bacterial cultures by inspection of growth, bioluminescence and RZ reducing velocity under different growth conditions. The growth of strain ANT-2200 under high hydrostatic pressure conditions was slightly slower than that at atmosphere pressure, and both cultures reached stationary phase after about 10h incubation (Fig. 5a). However, the cultures at atmosphere pressure exhibited a second growth phase and attained maximal optical density of 1.32 at $24 \mathrm{~h}$ and remained the same after $50 \mathrm{~h}$ incubation (Fig. 5a). Consistently the bioluminescence increased during exponential growth phase 
and decreased in stationary growth phase, and the cultures at atmosphere pressure were more luminous than those under high hydrostatic pressure (Fig. 5b). Notably, the instantaneous RZ reducing velocity increased during the exponential growth phase and slowed down at the stationary phase, and those of the cultures at atmosphere pressure were higher than those under high pressure (Fig. 5c). Together these results showed that the instantaneous reducing velocity reflects truly the physiological state of cultures and is suitable for evaluating the marine bacterial metabolism. 
a

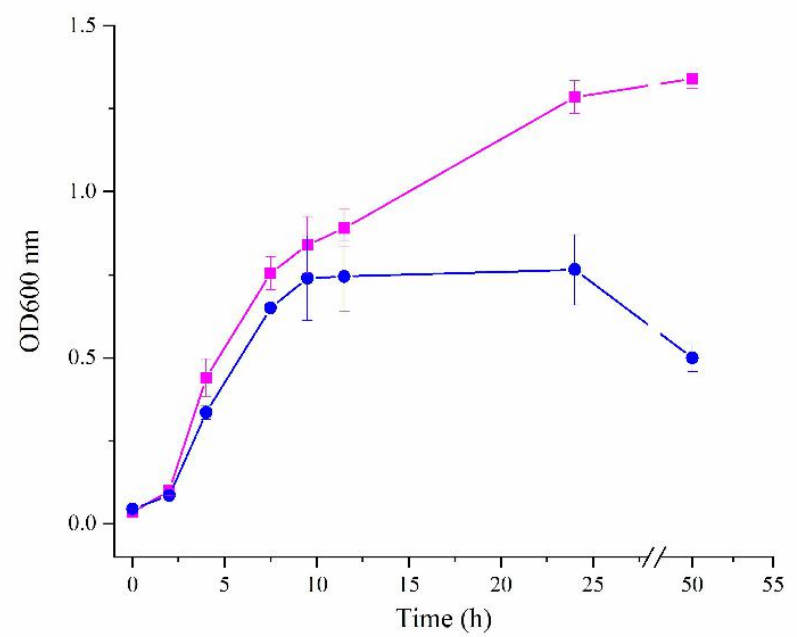

$\mathrm{b}$

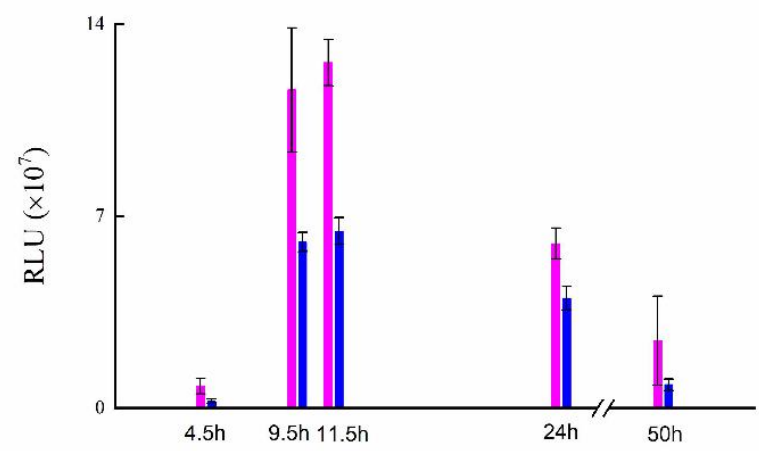

$\mathrm{c}$

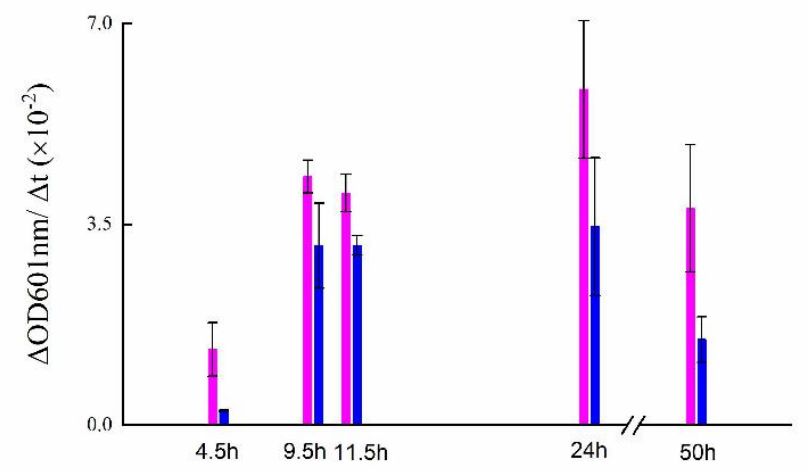

Fig. 5. Coherence of reducing capacity with growth and bioluminescence of ANT-2200 cultures. ANT-2200 were incubated at atmosphere pressure (red) or $20 \mathrm{MPa}$ (blue). The optical density at $600 \mathrm{~nm}$ (a), bioluminescence (b) and the calculated RZ reducing velocity (c) were taken at times as indicated.

\section{CONCLUSIONS}

Resazurin is a sensitive reducing capacity indicator. In this study we assessed the feasibility of using resazurin as reducing capacity indicator to analyze physiology state of marine luminous 
bacteria. RZ exhibits cytotoxicity at high concentrations and it should be cautious to use it directly in cultures. However, we found an obvious coherency between the reducing capacity of the cultures with their growth and bioluminescence. Resazurin can be used in physiological study of marine microorganisms.

\section{ACKNOWLEDGMENTS}

This work was supported by grant 2016YFC0302502, 2018YFC0309904 and 2016YFC0304905 from National Key R\&D Program of China, grants 91751202, 91751108 and 41806174 from NSFC, grants 2018YD01 and 2018YD02 from Sanya and grants from CNRS for LIA-MagMC.

\section{References}

Afkhami A, Safavi A, Massoumi A. 1991. Catalytic Spectrophotometric Determination of an Ultra-Trace Amount of Lead by Reduction of Resazurin by Sodium Sulfide. Analytical Letters, 24(9):1643-1655. https://doi.org/10.1080/00032719108053000.

Al Ali B, Garel M, Cuny P, et al. 2010. Luminous bacteria in the deep-sea waters near the ANTARES underwater neutrino telescope (Mediterranean Sea). Chemistry and Ecology, 26(1):57-72. https://doi.org/10.1080/02757540903513766.

Barnes S, Spenney J G. 1980. Stoichiometry of the nadh-oxidoreductase reaction for dehydrogenase determinations. Clinica Chimica Acta, 107:149-154. https://doi.org/10.1016/0009-8981(80)90442-8.

Bueno C, Villegas M L, Bertolotti S G, et al. 2002. The excited-state interaction of resazurin and resorufin with aminesin aqueous solutions. photophysics and photochemical reaction $\uparrow$ Photochemistry and Photobiology, 76(4), 385-390. https://doi.org/10.1562/0031-8655(2002)0760385TESIOR2.0.CO2.

Dunlap P V, Kita-Tsukamoto K. 2006. Luminous Bacteria The Prokaryotes. p. 863-892.

Jong D W D, Woodlief W G. 1977. Fluorimetric assay of tobacco leaf dehydrogenases with resazurin. Biochimica et Biophysica Acta, 484(2), 249-259. https://doi.org/10.1016/0005-2744(77)90081-X.

Kowaltowski A J. 2019. Strategies to detect mitochondrial oxidants. Redox Biol, 21, 101065. https://doi.org/10.1016/j.redox.2018.101065.

Martini S, Al Ali B, Garel M, et al. 2013. Effects of hydrostatic pressure on growth and luminescence of a moderately-piezophilic luminous bacteria Photobacterium phosphoreum ANT-2200. PLoS One, 8(6), 
e66580. https://doi.org/10.1371/journal.pone.0066580.

Neumann M G, Schmitt C C, Previtali C M, et al. 1996. Photoreduction of resazurin in the presence of aliphatic amines. Dyes and Pigments, 32(2), 93-99. https://doi.org/10.1016/0143-7208(96)00020-4.

Nijvipakul S, Wongratana J, Suadee C, et al. 2008. LuxG is a functioning flavin reductase for bacterial luminescence. J Bacteriol, 190(5). https://doi.org/1531-1538. 10.1128/JB.01660-07.

Porcal G V, Altamirano M S, Bertolotti S G, et al. 2011. Organized media effect on the photochemical deoxygenation of resazurin in the presence of triethanolamine. Journal of Photochemistry and Photobiology A: Chemistry, 219(1), 62-66. https://doi.org/10.1016/j.jphotochem.2011.01.018.

Rampersad S N. 2012. Multiple applications of Alamar Blue as an indicator of metabolic function and cellular health in cell viability bioassays. Sensors (Basel), 12(9), 12347-12360. https://doi.org/10.3390/s120912347.

Safavi A, Afkhami A, Massoumi A. 1990. Spectrophotometric catalytic determination of ultra-trace amounts of selenium based on the reduction of resazurin by sulphide. Analytica Chimica Acta, 232, 351-356. https://doi.org/10.1016/S0003-2670(00)81253-2.

Zhang S D, Santini C L, Zhang W J, et al. 2016. Genomic and physiological analysis reveals versatile metabolic capacity of deep-sea Photobacterium phosphoreum ANT-2200. Extremophiles, 20(3). https://doi.org/301-310. 10.1007/s00792-016-0822-1.

Zhao B, Ranguelova K, Jiang J, et al. 2011. Studies on the photosensitized reduction of resorufin and implications for the detection of oxidative stress with Amplex Red. Free Radic Biol Med, 51(1), 153-159. https://doi.org/10.1016/j.freeradbiomed.2011.03.016. 